\section{Cetoacidosis diabética: Casuística 2008-2012, epidemiología y fisiopatología}

\author{
PABLO OLMOS ${ }^{1}$, ANÍBAL DONOSO $^{1}$, JUAN PABLO ARAB $^{\mathrm{a}}$, \\ IAN NIKLITSCHEK ${ }^{\mathrm{b}}$, NICOLÁS MERTENS $^{\mathrm{b}}$, ELIZABETH ARCE $^{2, \mathrm{c}}$, \\ ROSARIO LEMUS ${ }^{2, \mathrm{c}}$, VALENTINA SERRANO ${ }^{1}$, BRUNO GRASSI $^{\mathrm{d}}$, \\ KRISTEL STRODTHOFF ${ }^{\mathrm{d}}$, EDUARDO ABBOTT ${ }^{2}$, \\ ANDRÉS AIZMAN², MARÍA VERÓNICA GONZÁLEZ
}

\section{Treatment of diabetic ketoacidosis using 2009 American Diabetes Association guidelines}

Background: During 2009, new guidelines for the treatment of diabetic ketoacidosis were published by the American Diabetes Association. Aim: To assess the impact of new treatment guidelines on the evolution of patients treated for diabetic ketoacidosis (KAD). Patients and Methods: Anonymous data was obtained from computational medical records of patients treated for KAD at our institution two years before ("Traditional Protocol") and TWO years after ("ADA-2009 Protocol") the publication of the 2009 American Diabetes Association (ADA) KAD guidelines. Results: Twenty three patients aged $36.5 \pm 15.1$ years were treated with the traditional method and 23 patients aged $44.4 \pm 21.1$ years were treated following 2009 ADA guidelines. Among patients treated with the traditional protocol and treated following ADA 2009 guidelines, the diabetes type 1/type 2 ratio was18/5 and 19/16 respectively $(p=N S)$, the glycosylated hemoglobin on admission was $12.6 \pm 2.5$ and $14.3 \pm 2.7 \%$ respectively $(p=0.03)$, minimal blood $p H$ was $7.15 \pm 0.14$ and $7.19 \pm 0.09$ respectively $(p=N S)$, bicarbonate was required in seven and no patient respectively $(p=0.01)$, hypokalemia $<3.5 \mathrm{mEq} / \mathrm{L}$ occurred in 78.2 and $48.5 \%$ of patients $(p=0.03)$, the lapse until resolution was $28.7 \pm 28.0$ and $28.8 \pm 20.6$ hours ( $p=N S$ ). Only one patient, treated following ADA 2009 guidelines, died. Conclusions: Introduction of the ADA-2009 protocol for the treatment of KAD resulted in decrease in the use of intravenous bicarbonate and a reduction in the incidence of hypokalemia. There was no impact neither in the lapse until resolution or lethality.

(Rev Med Chile 2014; 142: 1267-1274)

Key words: Diabetes mellitus; Diabetic Ketoacidosis.

\author{
'Departamento de Nutrición, \\ Diabetes y Metabolismo Facultad \\ de Medicina. \\ 2Departamento de Medicina \\ Interna. Hospital Clínico de la \\ Pontificia Universidad Católica \\ de Chile. \\ aResidente de Gastroenterología, \\ Facultad de Medicina, Pontificia \\ Universidad Católica de Chile. \\ bAlumno-Ayudante de Medicina, \\ Facultad de Medicina, Pontificia \\ Universidad Católica de Chile, \\ Santiago de Chile. \\ 'Enfermera Universitaria. \\ ${ }^{\mathrm{d}}$ Residente de Diabetes $\mathrm{y}$ \\ Nutrición. Facultad de Medicina, \\ Pontificia Universidad Católica de \\ Chile, Santiago de Chile. \\ eResidente de Radiología. \\ Facultad de Medicina, Pontificia \\ Universidad Católica de Chile, \\ Santiago de Chile. \\ Recibido el 24 abril de 2014, \\ aceptado el 26 de septiembre \\ de 2014. \\ Correspondencia a: \\ Dr. Pablo R. Olmos \\ Departamento de Nutrición, \\ Diabetes y Metabolismo \\ Facultad de Medicina, Pontificia \\ Universidad Católica de Chile \\ Avenida Libertador Bernardo \\ O'Higgins 340, Santiago, Chile. \\ Teléfonos: 56-2-23543863 y 56- \\ 2-23543862 \\ Fax: 56-2-26338298 \\ polmos@med.puc.cl
}

L a cetoacidosis diabética (KAD), descrita en Alemania por A. Kussmaul en $1874^{1}$, y por los siguientes cincuenta años ${ }^{2}$, fue responsable de más de dos tercios de los fallecimientos de pacientes diabéticos. A partir del descubrimiento y rápida introducción de la insulina luego que ésta fuera aislada por Banting y Best en $1921^{3}$, la mortalidad específica por KAD descendió brus- camente, para estabilizarse y mantenerse hasta la actualidad en cifras del orden de $4 \%{ }^{4-8}$. Por otro lado, la letalidad de los episodios de $\mathrm{KAD}$, que se mantenía en $100 \%$ entre 1874 y $1901^{1,2}$, descendió gradualmente durante el siglo 20 en la medida que a la introducción de la insulina se sumaban, uno tras otro, grandes progresos en hidratación intravenosa, medición de electrolitos y masifica- 
ción de laboratorios clínicos hospitalarios ${ }^{9-19}$. En la actualidad, la letalidad de la KAD es del orden de $5 \%$, y gran parte de las personas que fallecen lo hacen a consecuencia de alguna enfermedad desencadenante, y no por la KAD misma ${ }^{19}$. Por esta razón, se están haciendo esfuerzos por reducir las complicaciones no letales de la KAD, como el edema cerebral, la hipokalemia y la hipofosfemia.

En el año 2009, Kitabachi y cols. llevaron a cabo la iniciativa de sistematizar gráficamente el tratamiento de la KAD, publicándolo en Diabetes Care, revista de la American Diabetes Association $(\mathrm{ADA})^{17}$. Estas normas de tratamiento, llamadas por esta razón “ADA 2009", fueron adoptadas por las unidades de terapia intermedia e intensiva de nuestra institución el mismo año 2009, y siguen vigentes hasta ahora.

Nuestro objetivo fue comparar la evolución de las KAD los dos años previos (2007-2008), con los dos años posteriores (2010-2011) a la adopción de estas normas, evaluando el impacto de éstas sobre las complicaciones no letales de la KAD.

\section{Pacientes y Método}

Mediante una dispensa formal de consentimiento informado, entregada por el Comité de Ética de nuestra institución, obtuvimos los datos anónimos de todas las KAD tratadas en nuestro Hospital Clínico en dos períodos: entre el $1^{\circ}$ de enero de 2007 y 31 de diciembre de 2008 ("Protocolo Tradicional", previo a normas ADA-2009), y entre el $1^{\circ}$ de enero de 2010 y el 31 de diciembre de 2011 ("Protocolo ADA-2009").

Los datos tabulados provienen de dos fuentes: a) La historia clínica, tanto médica como de enfermería, y b) El registro computacional de exámenes de laboratorio de nuestra institución, que incluye la fecha y la hora precisa de la toma de muestra.

El análisis estadístico incluyó pruebas " $\mathrm{t}$ " de Student para muestras independientes, pruebas de $\chi^{2}$ (o test "exacto" de Fisher) para variables categóricas. Los datos de los pacientes fueron tabulados en el programa PASW Statistics $\mathrm{N}^{\circ} 18$ (version 18.0.0, July 30th, 2009; (C) 2003-2007 Polar Engineering Consulting, USA). Las regresiones no lineales fueron exponenciales de exponente negativo de segundo orden, y se llevaron a cabo en el programa GraphPad Prism 5.0 for Windows (@1992-2007 GraphPad Software, Inc., USA). Los valores se expresaron en media aritmética más/ menos una desviación estándar. Consideramos como significativo un valor de "p" inferior a 0,05 para todas las pruebas estadísticas. No se llevó a cabo la corrección de Bonferroni para comparaciones múltiples, de acuerdo a las recomendaciones publicadas por Perneger TV ${ }^{20}$.

\section{Resultados}

La Tabla 1 muestra los datos demográficos de ambos grupos de pacientes. El grupo con Protocolo Tradicional tuvo una mayor proporción de pacientes con KAD por debut de diabetes que el grupo sometido a Protocolo ADA-2009, siendo en estos últimos la "no adherencia" a tratamiento de una diabetes diagnosticada previamente la causa desencadenante de KAD más frecuente. Las demás variables demográficas (tipo de diabetes, edad, género, creatinina) fueron similares en ambos grupos.

La Tabla 2 muestra las características clínicas de ambos grupos al momento del diagnóstico de la KAD e inicio de tratamiento son similares, incluyendo glicemia, osmolaridad plasmática, electrolitos, función renal y ácido-base.

En el grupo de Protocolo Tradicional, 3 de los 7 pacientes $(42,8 \%)$ que recibieron bicarbonato endovenoso desarrollaron alcalosis metabólica. En cambio, en el grupo Protocolo ADA 2009, ningún paciente recibió bicarbonato endovenoso, y ninguno $(0,0 \%)$ desarrolló alcalosis metabólica (Tablas 3 y 4). Entre ambos períodos, los casos de hipokalemia $(<3,5 \mathrm{mEq} / \mathrm{L})$ bajaron de $78,2 \%$ a 48,5\%, y las hipokalemias leves, entre 3,0 y 3,4 $\mathrm{mEq} / \mathrm{L}$, bajaron de $47,8 \%$ a $22,8 \%$ (Tabla 4 ). Es importante recalcar que si bien las hipokalemias graves, con potasio menor que $3,0 \mathrm{mEq} / \mathrm{L}$ fueron similares en ambos períodos, en el primero de ellos (2007-2008), sólo uno de 15 (6,7\%) pacientes que no recibieron bicarbonato endovenoso tuvo un potasio mínimo de $2,8 \mathrm{mEq} / \mathrm{L}$ o menos, y tres de $4(75 \%)$ de aquellos que sí recibieron tratamiento con bicarbonato presentaron ese grado de hipokalemia ( $(2$ de Parsons $=4,54, \mathrm{p}=0,033)$.

Combinando los resultados de Tablas 3 y 4, vemos que las hipokalemias en general $(<3,5$ $\mathrm{mEq} / \mathrm{L})$, y las graves en particular $(<3,0 \mathrm{mEq} / \mathrm{L})$ se concentran significativamente en el primer período (2007-2008), precisamente allí donde se concentra la totalidad de pacientes tratados con bicarbonato intravenoso ( 7 de 23 pacientes, 
Tabla 1. Características demográficas de los pacientes KAD

\begin{tabular}{|lccc|}
\hline Variable & $\begin{array}{c}\text { Protocolo } \\
\text { tradicional } \\
\text { (n = 23) }\end{array}$ & $\begin{array}{c}\text { Protocolo } \\
\text { ADA 2009 } \\
\text { (n = 35) }\end{array}$ & p \\
Período histórico de la hospitalización & $2007-2008$ & $2010-2011$ & - \\
Tipo de diabetes (DM1/DM2) & $18 / 5$ & $19 / 16$ & 0,094 \\
Patología psiquiátrica o adicción (sí/no) & $8 / 15$ & $5 / 30$ & 0,107 \\
Género (mujer/hombre) & $15 / 8$ & $22 / 13$ & 1,0 \\
Debut de diabetes mellitus (sí/no) & $12 / 11$ & $7 / 28$ & $\mathbf{0 , 0 1 2}$ \\
Edad (años) & $36,5 \pm 15,1$ & $44,4 \pm 21,1$ & 0,13 \\
HbA1c (\%) al ingreso & $12,6 \pm 2,5$ & $14,3 \pm 2,7$ & $\mathbf{0 , 0 3 1}$ \\
Creatinina (mg/dL) previa al ingreso & $0,74 \pm 0,04$ & $0,94 \pm 0,34$ & 0,329 \\
Patología desencadenante: infección/no-adherencia/debut/otros & $5 / 4 / 12 / 2$ & $8 / 18 / 7 / 2$ & $\mathbf{0 , 0 2 3 1}$ \\
\hline
\end{tabular}

Tabla 2. Características al ingreso de los pacientes KAD

\begin{tabular}{|c|c|c|c|}
\hline Variable & $\begin{array}{l}\text { Protocolo tradicional } \\
\qquad(n=23)\end{array}$ & $\begin{array}{l}\text { Protocolo ADA } 2009 \\
(n=35)\end{array}$ & $\mathbf{p}$ \\
\hline Período histórico de la hospitalización & $2007-2008$ & 2010-2011 & - \\
\hline $1^{\circ}$ glicemia $(\mathrm{mg} / \mathrm{dL})$ & $420,7 \pm 213,8$ & $384,1 \pm 155,6$ & 0,474 \\
\hline Osmolaridad plasmática (mOsm/Kg) & $300,9 \pm 13,7$ & $297,3 \pm 11,7$ & 0,319 \\
\hline $1^{\circ}$ potasio sérico $(\mathrm{mEq} / \mathrm{L})$ & $4,2 \pm 0,77$ & $4,6 \pm 1,12$ & 0,132 \\
\hline Hipokalemia/normokalemia/hiperkalemia & $5 / 15 / 3$ & $3 / 21 / 11$ & 0,153 \\
\hline $1^{\circ}$ sodio sérico (mEq/L) & $135,5 \pm 6,9$ & $135,0 \pm 4,8$ & 0,774 \\
\hline $1^{\circ}$ cloro sérico (mEq/L) & $98,8 \pm 13,2$ & $99,1 \pm 8,0$ & 0,904 \\
\hline $1^{\circ}$ bicarbonato sérico (mEq/L) & $10,0 \pm 4,8$ & $12,9 \pm 6,0$ & 0,061 \\
\hline $1^{\circ}$ brecha aniónica (Anion Gap, mEq/L) & $26,6 \pm 12,3$ & $22,9 \pm 6,7$ & 0,163 \\
\hline $1^{\circ}$ nitrógeno ureico sérico $(\mathrm{mg} / \mathrm{dL})$ & $18,6 \pm 11,0$ & $23,2 \pm 16,3$ & 0,243 \\
\hline $1^{\circ}$ fósforo sérico $(\mathrm{mg} / \mathrm{dL})$ & $3,9 \pm 2,8$ & $3,2 \pm 1,5$ & 0,321 \\
\hline $1^{\circ}$ creatinina sérica $(\mathrm{mg} / \mathrm{dL})$ & $1,08 \pm 0,4$ & $1,23 \pm 0,7$ & 0,389 \\
\hline $1^{\circ}$ proteína $\mathrm{C}$ reactiva $(\mathrm{mg} / \mathrm{dL})$ & $5,6 \pm 11,3$ & $6,27 \pm 11,2$ & 0,846 \\
\hline $1^{\circ}$ proteína $C$ reactiva $(>1,0 / \leq 1,0 \mathrm{mg} / \mathrm{dL})$ & $12 / 11$ & $21 / 14$ & 0,597 \\
\hline
\end{tabular}

contra cero en el período siguiente), y también el $\mathrm{pH}$ máximo es mayor $(7,38 \pm 0,05$, contra $7,34 \pm$ 0,02 en el período siguiente).

Llama la atención en la Tabla 3, que el potasio alcanza su valor mínimo bastante tarde en relación al momento de la primera glicemia, 22,6 \pm $17,1 \mathrm{~h}$ en el primer período, y 27,4 $\pm 30,7 \mathrm{~h}$ en el segundo $(\mathrm{p}=\mathrm{NS})$.
En la Tabla 5 se observa que la duración de la estadía en UCI/UTI fue similar en ambos grupos, lo mismo que la función renal al egreso. También en ambos grupos la mayoría de los pacientes quedaron con terapia insulínica al egreso: 16/23 $(69,5 \%)$ en el grupo de Protocolo Tradicional, y 25/35 (71,4\%) en el grupo de Protocolo ADA 2009.

Finalmente, la Tabla 6 muestra los resultados 
Tabla 3. Evolución durante la hospitalización en unidad de intensivo o intermedio de los pacientes KAD

\begin{tabular}{|c|c|c|c|}
\hline Variable & $\begin{array}{l}\text { Protocolo tradicional } \\
\qquad(n=23)\end{array}$ & $\begin{array}{l}\text { Protocolo ADA } 2009 \\
(n=35)\end{array}$ & $\mathbf{p}$ \\
\hline Período histórico de la hospitalización & $2007-2008$ & $2010-2011$ & - \\
\hline Potasio mínimo (mEq/L) & $3,2 \pm 0,41$ & $3,46 \pm 1,04$ & 0,183 \\
\hline Potasio mínimo (horas desde $1^{\circ}$ glicemia) & $22,6 \pm 17,1$ & $27,4 \pm 30,7$ & 0,467 \\
\hline Bicarbonato mínimo (mEq/L) & $8,59 \pm 4,41$ & $11,38 \pm 4,0$ & 0,025 \\
\hline Bicarbonato mínimo (horas desde $1^{\circ}$ glicemia) & $6,21 \pm 11,2$ & $4,06 \pm 10,0$ & 0,461 \\
\hline pH mínimo & $7,15 \pm 0,14$ & $7,19 \pm 0,09$ & 0,241 \\
\hline $\mathrm{pH}$ mínimo (horas desde $1^{\circ}$ glicemia) & $10,14 \pm 19,6$ & $3,03 \pm 11,6$ & 0,154 \\
\hline $\mathrm{pH}<7,1$ (sí/no) & $7 / 16$ & $5 / 30$ & 0,189 \\
\hline $\mathrm{pH}<6,9$ (sí/no) & $1 / 22$ & $0 / 35$ & 0,397 \\
\hline Terapia con bicarbonato intravenoso (si/no) & $7 / 16$ & $0 / 35$ & 0,001 \\
\hline Potasio máximo (mEq/L) & $4,60 \pm 0,68$ & $4,86 \pm 0,91$ & 0,233 \\
\hline Potasio máximo (horas desde $1^{\circ}$ glicemia) & $45,5 \pm 63,7$ & $21,6 \pm 37,7$ & 0,117 \\
\hline Bicarbonato máximo (mEq/L) & $23,04 \pm 2,9$ & $22,9 \pm 4,7$ & 0,911 \\
\hline Horas desde $1^{\circ}$ glicemia hasta bicarbonato máx & $54,4 \pm 47,1$ & $46,3 \pm 29,0$ & 0,467 \\
\hline pH máximo & $7,38 \pm 0,05$ & $7,34 \pm 0,02$ & 0,01 \\
\hline $\mathrm{pH}$ máximo (horas desde $1^{\circ}$ glicemia) & $44,8 \pm 35,2$ & $25,4 \pm 20,4$ & 0,033 \\
\hline Velocidad descenso de glicemia ([mg/dL]/h) & $15,6 \pm 10,7$ & $11,4 \pm 10,5$ & 0,332 \\
\hline Tiempo desde ingreso hasta resolución (horas)a & $28,7 \pm 28,0$ & $28,8 \pm 20,6$ & 0,995 \\
\hline
\end{tabular}

${ }^{a}$ Criterios de resolución: $\mathrm{pH} \geq 7,35$, glicemia 200 mg/dL.

Tabla 4. Complicaciones y Letalidad en unidad de intensivo o intermedio de los pacientes KAD

\begin{tabular}{|c|c|c|c|}
\hline Variable & $\begin{array}{l}\text { Protocolo tradicional } \\
\qquad(n=23)\end{array}$ & $\begin{array}{l}\text { Protocolo ADA } 2009 \\
(n=35)\end{array}$ & $\mathbf{p}$ \\
\hline Período histórico de la hospitalización & $2007-2008$ & $2010-2011$ & - \\
\hline Al menos una glicemia $<70 \mathrm{mg} / \mathrm{dL}$ & $4 / 23$ & $10 / 35$ & 0,233 \\
\hline Al menos una glicemia $<50 \mathrm{mg} / \mathrm{dL}$ & $1 / 23$ & $5 / 35$ & 0,211 \\
\hline Hipokalemia < 3,5 mEq/L & $18 / 23(78,2 \%)$ & $17 / 35(48,5 \%)$ & 0,031 \\
\hline Hipokalemia 3,0-3,4 mEq/L & $11 / 23(47,8 \%)$ & $8 / 35(22,8 \%)$ & 0,034 \\
\hline Hipokalemia < 3,0 mEq/L & $7 / 23(30,4 \%)$ & $9 / 35(25,7 \%)$ & 0,459 \\
\hline Alcalosis metabólica (pH > 7,45) & $3 / 23(13,0 \%)$ & $0 / 35(0,0 \%)$ & 0,084 \\
\hline Edema cerebral & $1 / 23(4,3 \%)$ & $0 / 35(0,0 \%)$ & 0,386 \\
\hline Letalidad de la KAD & $0 / 23(0,0 \%)$ & $1 / 35(2,85 \%)$ & 0,386 \\
\hline
\end{tabular}

de la investigación de los datos registrados en el sistema computacional de la red de salud de nuestra institución, con vistas a determinar la situación clínica de cada paciente el año 2013. Esta información fue posible de obtener en 15 de 23 pacientes $(65,2 \%)$ en el grupo de Protocolo Tradicional, y en 17 de $35(48,5 \%)$ en el grupo de Protocolo ADA 2009. El tiempo pasado entre la KAD y la información obtenida fue de $57,1 \pm$ 6,8 meses y 20,4 \pm 6,3 meses, respectivamente. En esta etapa, ambos grupos tuvieron hemoglobina glicosilada similar, de 7,93 $\pm 1,3 \%$ y $8,0 \pm 1,38 \%$, respectivamente. Las tasas de recaída de KAD fueron $4,34 \%$ y $5,71 \%$. 
Cetoacidosis diabética - P. Olmos et al

Tabla 5. Estado al egreso de unidad de intensivo o intermedio de los pacientes KAD

\begin{tabular}{|lccc|}
\hline Variable & $\begin{array}{c}\text { Protocolo tradicional } \\
(\mathbf{n}=\mathbf{2 3})\end{array}$ & $\begin{array}{c}\text { Protocolo ADA 2009 } \\
\text { (n = 35) }\end{array}$ & p \\
\hline Período histórico de la hospitalización & $2007-2008$ & $2010-2011$ & - \\
Estadía en UCI/UTI (días) & $7,57 \pm 3,5$ & $8,2 \pm 8,9$ & 0,708 \\
Dieta/HO/Insulina Convencional/Insulina Intensificada & $0 / 2 / 3 / 16$ & $3 / 5 / 0 / 25$ & 0,172 \\
Creatinina sérica (mg/dL) & $0,62 \pm 0,2$ & $0,64 \pm 0,1$ & 0,747 \\
Nitrógeno Ureico Sanguíneo (BUN, mg/dL) & $12,5 \pm 9,3$ & $12,15 \pm 6,3$ & 0,892 \\
Proteína C Reactiva (mg/dL) & $2,7 \pm 2,9$ & $1,5 \pm 2,1$ & 0,215 \\
\hline
\end{tabular}

Tabla 6. Estado al seguimiento posterior meses después de egresar desde la unidad de intensivo o intermedio de los pacientes KAD

\begin{tabular}{|lccc|}
\hline Variable & $\begin{array}{c}\text { Protocolo tradicional } \\
(\mathbf{n}=\mathbf{2 3})\end{array}$ & $\begin{array}{c}\text { Protocolo ADA 2009 } \\
\text { (n = 35) }\end{array}$ & p \\
\hline Período histórico de la hospitalización & $2007-2008$ & $2010-2011$ & - \\
Información posterior obtenida (\%) & $15(65,2 \%)$ & $17(48,5 \%)$ & 0,164 \\
Momento de la información (meses post egreso) & $57,1 \pm 6,8$ & $20,4 \pm 6,3$ & $\mathbf{0 , 0 0 0}$ \\
Dieta/HO/Ins. Convencional/Ins. Intensificada & $0 / 1 / 2 / 14$ & $0 / 4 / 2 / 12$ & 0,382 \\
Hemoglobina Glicosilada más reciente (por ciento) & $7,93 \pm 1,3$ & $8,0 \pm 1,38$ & 0,775 \\
Nueva Ketoacidosis en los meses post egreso (\%) & $1(4,34 \%)$ & $2(5,71 \%)$ & 0,562 \\
Muerte & $0(0,0 \%)$ & $1(2,85 \%)$ & 1,0 \\
\hline
\end{tabular}

$\mathrm{HO}=$ Hipoglicemiantes orales. Ins. $=$ Insulina.

\section{Discusión}

Nuestros datos muestran que, dos años después de la introducción de las normas ADA 2009 para el tratamiento de la cetoacidosis diabética, la frecuencia de hipokalemia, tanto en general como grave $(<3,0 \mathrm{mEq} / \mathrm{L})$ se redujo significativamente. Este fenómeno ocurre en paralelo con (y a consecuencia de) una drástica reducción en la frecuencia de uso de bicarbonato intravenoso, con lo que también se redujo a cero la frecuencia de alcalosis metabólica en el grupo de Protocolo ADA 2009.

En efecto, la fisiopatología de la KAD nos muestra que la poliuria que la precede lleva a una pérdida importante de iones de potasio, que muchas veces no se nota al ingreso del paciente al hospital, porque la acidosis metabólica obliga a los iones hidrógeno $(\mathrm{H}+)$ a entrar en las células, intercambiándose con iones de potasio $(\mathrm{K}+)$ que salen de éstas hacia el líquido extracelular. En otras palabras, al ingreso al hospital, el paciente con KAD está en una situación de "déficit de potasio corporal total con kalemia normal". Este déficit de potasio alcanza a 3-15 $\mathrm{mEq}$ por $\mathrm{Kg}$ de peso del paciente ${ }^{22}$. Una vez iniciado el tratamiento del paciente sólo con insulina e hidratación, por un lado la insulina obliga al potasio a entrar a las células, y por otro, la producción hepática de cetoácidos cesa completamente, y a medida que éstos son metabolizados en tejidos extrahepáticos, el pH sanguíneo comienza a subir, acercándose a lo normal. En este proceso, aun sin mediar administración de bicarbonato, se reducen tanto la entrada de $\mathrm{H}+$ a las células como la salida de $\mathrm{K}+$ hacia el líquido extracelular. Como resultado, el potasio plasmático comienza a disminuir gradualmente, fenómeno que ocurrirá siempre, a no ser que el tratamiento de la KAD incluya cierta cantidad de cloruro de potasio (20-30 $\mathrm{mEq})$ por 
cada litro de suero administrado. Ahora bien, si a esta situación sumamos la administración de bicarbonato exógeno, el alza brusca de $\mathrm{pH}$ sanguíneo, que puede exceder el máximo normal, produce una inversión del intercambio hidrógeno/ potasio en las células, con la consiguiente salida de $\mathrm{H}+$ hacia el extracelular y entrada de $\mathrm{K}+$ desde el líquido extracelular al interior de las células. El resultado es una hipokalemia grave y súbita, que puede producir arritmia refractaria, paro cardiaco y muerte ${ }^{21}$.

Es importante recalcar que la hipokalemia puede presentarse al momento del diagnóstico de $\mathrm{KAD}$, es decir, antes de iniciar el tratamiento, lo que ocurrió en nuestros pacientes en $21,7 \%$ en el grupo convencional ( 5 de 23 ) y en $8,5 \%$ en el grupo tratado según protocolo ADA-2009 (3 de 35). En 2012, Arora y cols. ${ }^{22}$ comunicaron una serie de $54 \mathrm{KAD}$ tratadas en Canadá según protocolo ADA 2009, donde la frecuencia de hipokalemia al ingreso fue de $5,6 \%$. Estas cifras son lo suficientemente importantes como para que nosotros estemos de acuerdo con los autores de ese artículo y con las normas ADA-2009 en el sentido de obtener una medición de potasio plasmático antes de iniciar tratamiento con insulina en pacientes con KAD.

Otros grupos han comunicado la reducción significativa de la hipokalemia durante el tratamiento al aplicar las normas ADA-2009. HYPERLINK "http://www.sciencedirect.com/science/

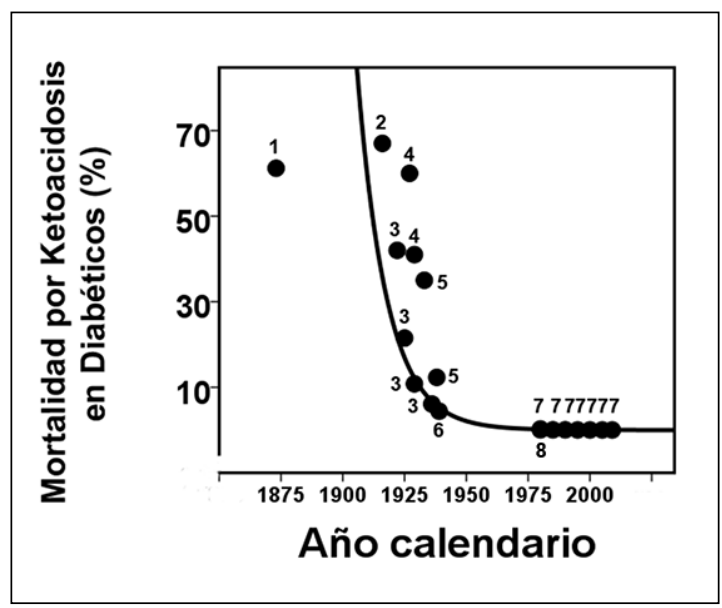

Figura 1. Mortalidad específica por ketoacidosis en pacientes diabéticos en el mundo, entre los años 1874 y 2012 ( $r=$ 0,$962 ; p=0,000$ ). El número arábigo sobre cada punto del gráfico corresponde a la referencia bibliográfica.
article/pii/S016882271400031X” Thuzar y cols ${ }^{23}$, en Australia, comunicaron una reducción de la hipokalemia desde $52 \%$ a $28 \%$, cifras similares a la reducción de hipokalemia moderada observada en nuestros pacientes desde $47,8 \%$ a $22,8 \%$ al pasar de terapia convencional a tratamiento con un protocolo similar al ADA-2009. HYPERLINK "http://www.sciencedirect.com/science/article/ pii/S016882271400031X” Thuzar y cols ${ }^{23}$, también comunicaron una disminución significativa de las hipoglicemias desde $28 \%$ a $8,6 \%$ al aplicar las normas ADA-2009. En cambio, en nuestros pacientes no hubo cambio significativo en la frecuencia de hipoglicemia (glicemia $<50 \mathrm{mg} / \mathrm{dL}$ ), que pasó de $4,3 \%(1 / 23)$ a $14,2 \%(5 / 35)$.

Aunque la mortalidad específica por cetoacidosis en la población diabética (Figura 1) ha disminuido desde cifras cercanas a $70 \%$ entre 1874 y 1920 , hasta $4 \%$ en la actualidad, resulta de capital importancia mantener la letalidad de esta complicación (Figura 2), lo más baja posible, es decir, menor que 5\%. Para ello se requiere que las unidades de tratamiento médico intermedio e intensivo se atengan a las normas más recientes, en este caso, las ADA-2009 (Figura 3), una de cuyas virtudes es, precisamente, la limitación estricta del uso de bicarbonato intravenoso, el que sólo deberá administrarse en caso de acidosis metabólica severa (es decir, con $\mathrm{pH}$ sanguíneo menor que $6,9)$. Otra virtud de esta norma es que posterga el

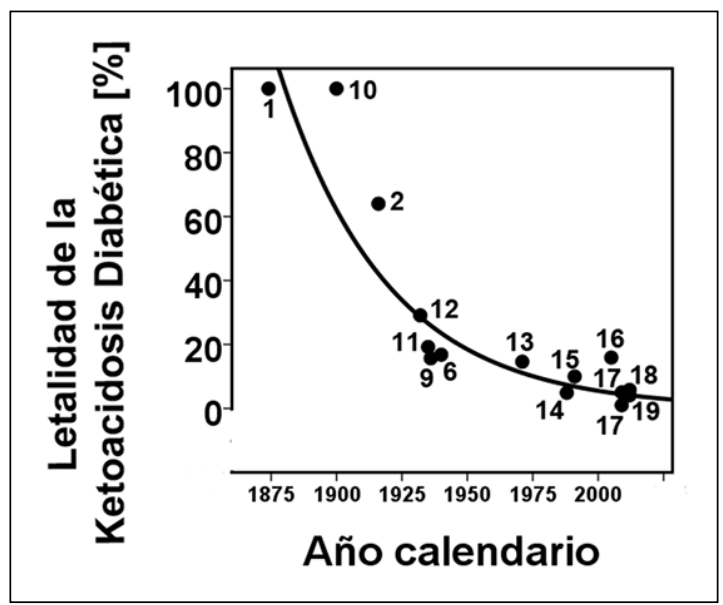

Figura 2. Letalidad de la ketoacidosis diabética en el mundo, entre los años 1875 y 2012 ( $r=0,879 ; p=0,000)$. El número arábigo sobre cada punto del gráfico corresponde a la referencia bibliográfica. 
HAGA una evaluacion inicial completa. MIDA glicemia capilar y cetonuria/cetonemia para confirmar hiperglicemia y cetonemia/cetonuria. INDIQUE un perfil bioquímico. INICIE hidratación EV con solución CINa al 0,9\% un litro por hora

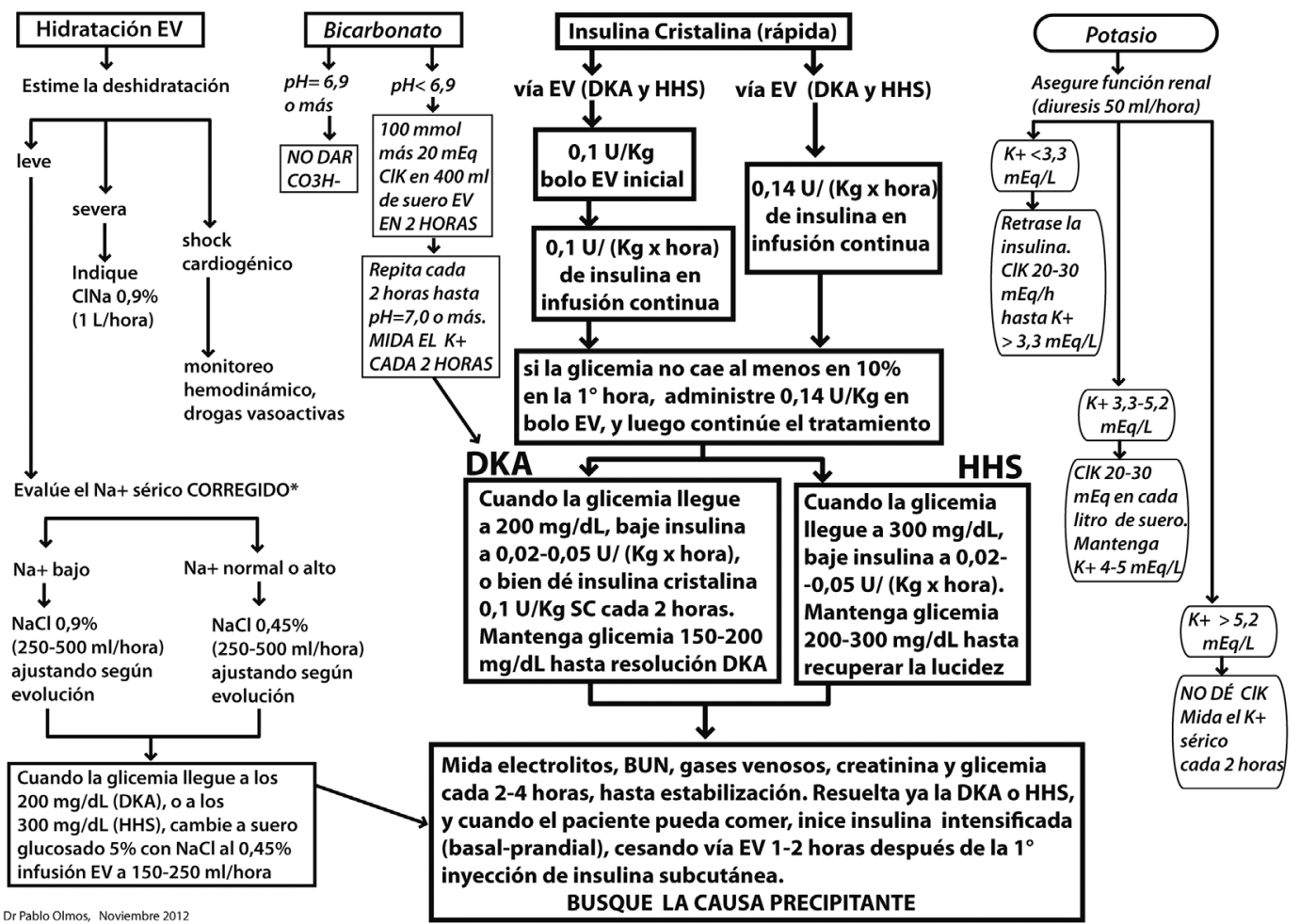

Figura 3. Flujograma que resume las normas de tratamiento de las "crisis hiperglicémicas" (DKA = ketoacidosis diabética por sus siglas en inglés o KAD y HHS = estado hiperglicémico hiperosmolar por sus siglas en inglés = coma hiperosmolar no ketósico), basado en (17). Abreviaturas: $\mathrm{EV}=$ endovenoso; $\mathrm{SC}=$ subcutáneo; $\mathrm{ClK}=$ cloruro de potasio; $\mathrm{CO} 3 \mathrm{H}=$ bicarbonato; $\mathrm{BUN}=$ nitrógeno ureico sanguíneo por sus siglas en inglés. $\mathrm{K}+=$ potasio. ${ }^{*} \mathrm{Na}+$ sérico corregido: Por cada $100 \mathrm{mg} / \mathrm{dL}$ de elevación de glicemia por sobre los $100 \mathrm{mg} / \mathrm{dL}$, debe sumarse 1,6 mEq/L a la concentración de $\mathrm{Na}+$ informada por el laboratorio ${ }^{17}$.

inicio de terapia con insulina cuando el potasio plasmático de ingreso en menor que $3,3 \mathrm{mEq} / \mathrm{L}^{17}$. En nuestros pacientes, aunque las frecuencias de hipokalemia de ingreso $(<3,5 \mathrm{mEq} / \mathrm{L})$ fueron $5 / 23$ $(21,7 \%)$ y $3 / 35(8,57 \%)$ en los protocolos tradicional y ADA-2009 respectivamente, en ningún caso el potasio de ingreso fue inferior a $3,3 \mathrm{mEq} / \mathrm{L}$, de modo que la única causa de hipokalemia durante el tratamiento de la KAD sería atribuible a la terapia misma, particularmente al uso de bicarbonato. En otras palabras, postulamos que la totalidad de la reducción de la frecuencia de hipokalemia que observamos al pasar de protocolo tradicional a ADA-2009 es atribuible a la limitación drástica en el uso de bicarbonato intravenoso.
En conclusión, la introducción de la norma ADA-2009 para tratamiento de la KAD en nuestra institución ha resultado en: uso menos frecuente de bicarbonato intravenoso, y menor incidencia de hipokalemia. No se ha registrado un impacto significativo en el tiempo de resolución ni en la letalidad, esta última dentro de rangos aceptados a nivel internacional (menos que 5\%).

\section{Referencias}

1. Kussmaul A. Zur Lehre von Diabetes Mellitus. Über eine eigentümliche Tosdesart bei Diabetischen, über Acetonämie, Glycerin-Behandlung des Diabetes und Einspirtzungen von Diastase in's Blut bei dieser Krankheit. 
Deutsches Archiv für Klinische Medizin 1874; 14: 1-46 (texto completo disponible en: http://www.bium.univparis5.fr/hist/medmedica/cote?epo0585).

2. Joslin EP. The Treatment of Diabetes Mellitus. With observations upon the disease based upon one thousand cases. 1916 por editorial Lea \& Febiger, New York, USA. Pp 56-7.

3. Marble A. Chapter XII, Diabetic Coma. En: Joslin EP, Root HF, White P, Marble A, The treatment of Diabetes Mellitus. 1937 by Lea \& Febiger, New York, USA. Pp 351-3.

4. Rabinowitch IM, Diabetic Coma and Diabetic Mortality Rates. Can Med Assoc J 1929; 21 (5): 583-6.

5. Rabinowitch IM, Fowler, Bensley. Ann Internal Med 1939; 12: 1403

6. Marble A. Chapter XII, Diabetic Coma. En: Joslin EP, Root HF, White P, Marble A, The treatment of Diabetes Mellitus. 1940 by Lea \& Febiger, New York, USA. Pp 366-7.

7. Department of Health and Human Services. Centers for Disease Control and Prevention. Crude and age-adjusted death rates for hyperglycemic crises and underlying cause per 100.000 diabetic population, United States, 1980-2009. http://www.cdc.gov/diabetes/statistics/mortalitydka/fRateDKADiabTotals.htm (page last reviewed May 10, 2012). Acceso el 9 de octubre de 2012.

8. Shenfield GM, Bhalla IP, Elton RA, Duncan LJ. Fatal coma in diabetes. Diabete Metab 1980; 6 (2): 151-5.

9. Baker TW. A clinical survey of one hundred and eight consecutive cases of diabetic coma. Arch Int Med 1936; 58 (3): 373-406 (citado x Joislin 1937).

10. Herter CA. The acid intoxication of diabetes in its relation to prognosis. J Exp Med 1901; 5 (6): 617-33 http:// www.ncbi.nlm.nih.gov/pmc2118025/ (acceso el 4 de octubre de 2012).

11. John HJ. Treatment of diabetic coma. Clinical lectura at Atlantic City session: JAMA 1935; 105: 587-92.

12. Bertram F. Pathogenese und prognose des coma diabeticum Volume 43 de Ergebnisse der Ineren Medzin und Kinderheilkunde. J. Springer 1932.

13. Prachar H, Brudener H, Nobis H, Korp W. Diabetic coma. A report of 752 cases treated between 1931 and
1973. MMW Munch Med Wochenschr 1975; 117 (16): 661-8.

14. Hamblin PS, Topliss DJ, Chosich N, Lording DW, Stockigt JR. Deaths associated with diabetic ketoacidosis and hyperosmolar coma. 1973-1988. Med J Aust 1989; 151 (8): 439-44.

15. Sajti I, Gymesi A, Hoffmann E, Czuczor J, Tomóczky J, Iványi J. Diabetic ketoacidosis (169 episodes in 131 patiernts in the course of 10 years). Orv Hetil 1991; 132 (15): 787-91.

16. Niaz Z, Razzaq A, Chaudhary UJ, Awais M, Yassen MA, Naseer I, et al. Mortality review of diabetic ketoacidosis in Mayo Hospital, Lahore-Pakistan. Biomedica 2005; 21 : 83-5.

17. Kitabachi AE, Umpierrez GE, Miles JM, Fisher JN. Hyperglycemic crises in adult patients with diabetes. Diabetes Care 2009; 32 (7): 1335-43.

18. Anthanont $\mathrm{P}$, Khawcharoenporn $\mathrm{T}$, Tharavanij T. Incidences and outcomes of hyperglycemic crises: a 5-year study in a tertiary care center in Thailand. J Med Assoc Thai 2012; 95 (8): 995-1002.

19. Barski L, Nevzorov R, Rabaev E, Jotkowitz A, HarmanBohem I, Zektser M, et al. Diabetic ketoacidosis: clinical characteristics, precipitating factors and outcome of care. Isr Med Assoc J 2012; 14 (5): 299-303.

20. Perneger TV. What's wrong with Bonferroni Adjustments. BMJ 1998; 316 (7139): 1236-8.doi: http:// dx.doi.org/10.1136/bmj.316.7139.1236 (Published 18 April 1998).

21. Blouin D. Too much of a good thing: Management of diabetic ketoacidosis in adults. Canadian Family Physician 2012; 58: 1099-100.

22. Arora S, Cheng D, Wyler B, Menchine M. Prevalence of hypokalemia in ED patients with diabetic ketoacidosis. American Journal of Emergency Medicine 2012; 30 (3): 481-4.

23. Thuzar M, Usman H. Malabu UH, Tisdell B, Sangla KS. Use of a standardised diabetic ketoacidosis management protocol improved clinical outcomes. Diab Res Clin Pract 2014. Epub ahead of print: http://dx.doi. org/10.1016/j.diabres.2014.01.016. 